\title{
Diseño y elaboración de experimentos demostrativos para la enseñanza de la física en el aula de clase
}

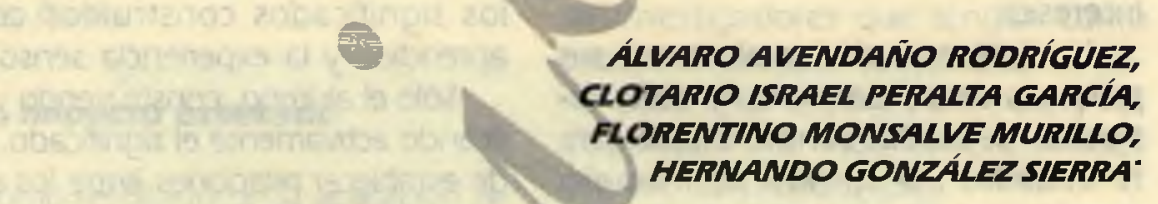

El presente artículo resume aspectos de la investigación "DISEÑO Y ELABORACIÓN DE EXPERIMENTOS DEMOSTRATIVOS PARA LA ENSEÑANZA DE LA FíSICA EN EL AULA DE CLASE" coordinada por profesores de la Facultad de Educación y de la Facultad de Ciencias Naturales y Exactas con la colaboración de siete estudiantes de la Facultad de Educación.

\section{Marco Teórico}

Se parte de reconocer la existencia, en los alumnos, de ideas, conceptos y teorías (ICT) antes de empezar el aprendizaje de las ciencias.

A menudo, este ICT aunque, es explicable dentro del mundo infantil, no coincide con el ICT de los hombres de ciencia y, lo indeseable, la mayor parte de él permanece sin ser influenciado durante el proceso curricular actual.

El desarrollo de conceptos depende de la acción de re-significar, por parte del alumno, el lenguaje del profesor. Hay que desarrollar un lenguaje que tenga el mismo significado para el alumno y para el profesor.

\subsection{Construir a partir de las ideas previas}

EI ICT desarrollado con anterioridad por los alumnos se expresa en términos particulares y formas diferentes de lenguaje. A las ideas se suele llegar con independencia de lenguaje que las soporta!

* Profesores de la Facultad de Ciencias Naturales y Exactas y de la Facultad de Educación de la Universidad Surcolombiana.

1 Osborne Roger y Peter Freyberg: (1991): "El aprendizaje de las Ciencias. Implicaciones de la ciencia de los alumnos". Universidad de Wickato, Narcea, S.A. Ediciones, 1991, p. 74. 


\section{6}

Universidad Surcolombiana

El ICT determina el desarrollo de nuevos conceptos, no siempre similares a los de los científicos, y esta influencia permanece a pesar de la enseñanza.

La inmadurez cognitiva, la poca experiencia y el uso inapropiado del lenguaje limitan, el proceso de pensamiento y de construcción de sentido y hasta el desarrollo de nuevos intereses.

Los intereses de los alumnos, sus procesos de pensamiento y su construcción de sentido son inexorablemente limitados por su nivel de madurez cognitiva, sus experiencias, su uso del lenguaje y su conocimiento y apropiación de las ideas de los demás.

Los niños, como los científicos, no rechazarán una idea a menos que tengan una alternativa disponible y atractiva que les sirva.

Una vez conocidas las ideas previas, es posible plantear secuencias de presentación alternativas que, considerando dichas ideas, lleven más fácil y directamente a los alumnos a aceptar una perspectiva científica.

Osborne $^{2}$ y su grupo, plantean 3 diferencias fundamentales entre el razonamiento de los niños y el de los científicos adultos:

Los niños, a diferencia de los científicos adultos, son limitados en el uso del razonamiento abstracto. Pueden aceptar varias explicaciones para el mismo acontecimiento, aunque sean contradictorias y tampoco tiene sentido verificar o no una idea para aceptarla o descartarla como científica.

\subsection{Construir aprendizaje}

Wittrock ${ }^{3}$ postula que el aprendizaje comprensivo parte de experiencias propias sensoriales. El dato seleccionado, es por sí mismo un significado inherente para el alumno. El hecho de escuchar las palabras del profesor no implica que esté percibiendo el significado del profesor, hay que contrastar los significados construidos con lo aprendido y la experiencia sensorial.

Sólo el alumno, construyendo y verificando activamente el significado, puede establecer relaciones entre los datos sensoriales y las ideas preexistentes.

El profesor, como ayudante del aprendizaje en el modelo generativo, cambia radicalmente el papel que desempeña: Conoce las ideas previas de sus alumnos, comprende sus formas de procesar la información y define los significados que quiere que obtengan sus alumnos; organiza el entorno de aprendizaje de modo que los alumnos tengan máximas probabilidades de generarlos por sí mismos, de modificar sus ideas existentes para asimilar las nuevas.

\section{Planteamiento del problema}

El ICT sobre los tópicos fundamentales de las ciencias de los alumnos de la región no es conocido y por ello es necesario investigarlo. Sin este conocimiento no es posible proponer alternativas pedagógicas que conduzcan a un ICT más inteligible, plausible y fructífero. 
El ICT es el punto de partida para organizar el conocimiento del área de Física, de manera que se produzca el cambio hacia el conocimiento científico de los fenómenos.

Sin conocer el ICT, no se podrán definir los medios y métodos más adecuados para lograr el cambio de las ideas, conceptos y teorías de los alumnos, por las ideas, conceptos y teorías de los físicos.

\section{Impacto esperado}

Como resultado de la aplicación de una propuesta alternativa para el aprendizaje de las Ciencias Naturales se espera un cambio conceptual en los alumnos de básicas que les permita en mayor número y calidad acceder a las oportunidades de continuar sus estudios con éxito en las diferentes universidades e institutos del mundo.

Para los alumnos del programa de Licenciatura en Ciencias Naturales, su participación en el proyecto les proveerá de un medio para desarrollar competencias investigativas, permitiendo, como futuros profesionales de la educación, prestar con mejor calidad el servicio educativo en el lugar de trabajo y específicamente en esta área.

Para los docentes en ejercicio, el desarrollo del proyecto, generará programas específicos de capacitación que reforzarán su compromiso en la generación del cambio necesario, para mejorar la calidad del aprendizaje.

Como resultado, se tendrá la formulación en los establecimientos de educación formal de la región surcolombiana en los cinco grados de educación primaria, los 4 grados de educación básica secundaria y los dos grados de educación media.

\section{Usuarios directos y potenciales de los resultados de la investigación}

Los beneficiarios directos de este proyecto están constituidos por los docentes que orientan el aprendizaje de las Ciencias Naturales en la región surcolombiana y sus alumnos. Potencialmente se beneficiarán los grupos de investigadores que tengan como propósito formular propuestas similares para otras regiones del país, y los docentes y alumnos comprometidos con dichas propuestas.

\section{Objetivos}

Identificar los conceptos, ideas y teorías más comunes que utilizan los estudiantes de los diferentes grados de educación básica primaria, básica secundaria y media vocacional, para referirse a temas fundamentales de la física, tales como movimiento, fuerza, energía, luz, calor, corriente eléctrica, etc.

Formular una propuesta curricular alternativa para el aprendizaje de la física en los diferentes grados de educación básica primaria, básica secundaria y media vocacional, que considere las ideas, conceptos y teorías previas de los alumnos y desarrolle estrategias adecuadas para que ellos construyan su conocimiento científico.

\section{Metodología}

Se inicia con la investigación del ICT de los alumnos de grado primero. Para cada tema fundamental se entrevistarán 40 alumnos. A partir de las entrevistas se elaborará un modelo de la forma como los estudiantes procesan la información. 
Paralelamente se definirán los significados físicos fundamentales. Estos significicađđôs sêrấn đesarrollados, acor= de con la forma como los alumnos procesan la información, en guías de trabajo para la clase. Estas guías se someterán a una prueba de convalidación y finalmente se organizará en la propuesta curricular alterna, que con- templará los recursos y actividades que perfilen el entorno de aprendizaje, acorde a lo expresado en el marco teórico.

Apenas se complete el ICT del grado primero, se empezará con el grado segundo y así, hasta terminar con el grado $11^{\circ}$ de educación media vocacional.

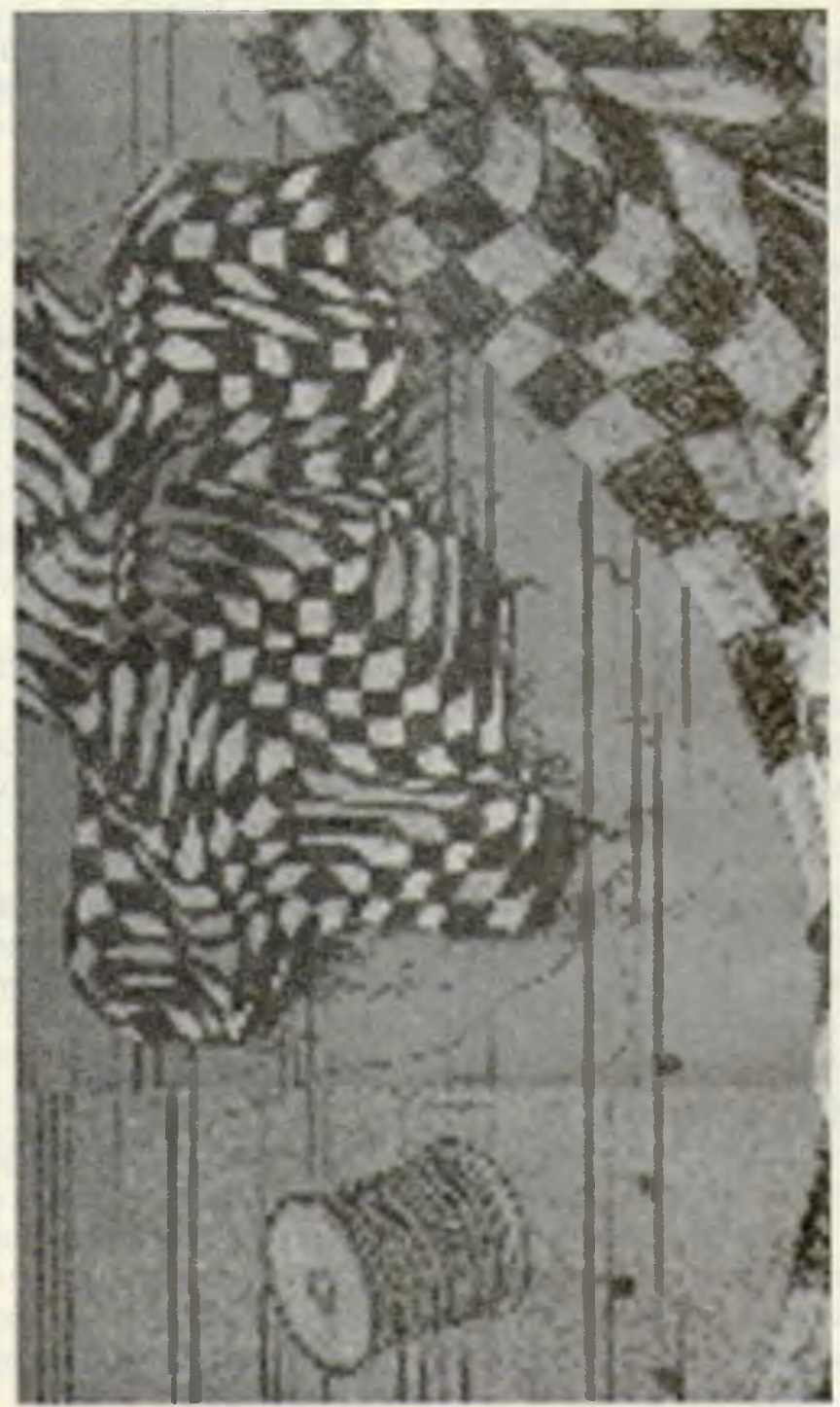




\section{9}

Paideia Surcolombiana

\section{REFERENCIAS BIBLIOGRÁFICAS}

1. AUSUBEL, D: "Psicología Educativa: Un punto de vista cognoscitivo": Trillas, México, 1976. P.IV.

2. BARNES, D. (1969), "Language, the learner and the school". Penguin books, Hammondsworth, Inglaterra.

3. EDWARDS, J. y MARLADN, P. (1982). "Student thinking in a secondary biology classroom". Research in Science Education, 12, 32-41

4. HERNÁNDEZ H., CARLOS A. Conferencia sobre el Estado del conocimiento en pedagogía de las ciencias, Colciencias, 2000.

5. HEUSON P. W. (1981): 'Aristotle: Alive and well in the classroom": Australian Science Teachers Journal, 27(3), 9-13 (a).

6. KELLY, G. A. (1969): “Ontological acceleration": The selected Papers of George Kelly. Wiley. N.Y

7. Osborne 1985. Marcea S.A. de Ediciones.

8. Osborne 1985. Marcea S.A. de Ediciones.

9. OSBORNE ROGER Y PETER FREYBERG: (1991): "El Aprendizaje de las Ciencias, Implicaciones de la ciencia de los alumnos": Universidad de Wickato, Narcea, S.A. Ediciones, 1991, p. 32.

10. OSBORNE, R.J. Y WITROCK, M.C. (1983): "Learning science: a generative process". Science Education, 67|4|, 489-508.

11. POSNER G. J.; STRIKE, A.; HEUSON, PW. Y GERTZOG W.A. (1982): “Accommodation of scientific conception: toward a theory of conceptual change": Science Education 66(2) $211-227$.

12. SOLOMON, J. (1980): "Teaching children in the laboratory". Croom - Helm. Londres. 1 WEST, L.H.T. y PINES, A. L. (1983): "How rational is rationality"? Science Education, $67(1), 37-39$.

13. WITTROCK, M.C. (1974), "Learning as a generative process". Educational Psychology, II, $87-95$. 